Forschende

Komplementärmedizin und

Klassische Naturheilkunde
Editorial

Forsch Komplementärmed Klass Naturheilkd 2001;8:253-254

\title{
Modellvorhaben Akupunktur: Mehr Chancen als Risiken
}

Gemäss Beschluss des Bundesausschusses der Ärzte und Krankenkassen vom 16.10.2000 soll zur weiteren Klärung der Frage, ob die Akupunktur eine Leistung der gesetzlichen Krankenkassen in Deutschland werden soll, ein Modellvorhaben «Akupunktur» durchgeführt werden. Die gesetzlichen Vorgaben nach $\S 63$ ff SGB V bestimmen als Ziel eines Modellvorhabens die Verbesserung der Qualität und der Wirtschaftlichkeit der Versorgung. Qualität umfasst hier neben der Qualität der ärztlichen Anwendung in erster Linie die Evaluation ihres therapeutischen Nutzens in Relation zum Risiko wie auch zu den Kosten. Für die nach § 65 SGB V verpflichtende wissenschaftliche Begleitung und Auswertung des Modellvorhabens wurden seitens des Bundesausschusses konkrete Empfehlungen veröffentlicht. Hierin werden explizit randomisierte und placebokontrollierte klinische Studien im Sinne biomedizinischer Forschung gefordert. Da der Bundesausschuss primär nach Lage der Evidenz vorhandener klinischer Studien entscheidet, ist diese Forderung folgerichtig. Gleichwohl schliesst das SGB V eine biomedizinische Forschung im Rahmen von Modellvorhaben aus - ein derzeit schwieriger Spagat.

Darüber hinaus sieht der Autor erheblichen Diskussionsbedarf darin, ob die Anforderungen an das Modellvorhaben mit diesem alleinigen methodischen Ansatz erfüllbar sind. Die gestellte Frage ist vielschichtig, und deshalb muss die wissenschaftliche Begleitung auf mehreren Ebenen der Beantwortung dienen. Neben dem traditionellen Wirksamkeitsnachweis (engl. efficacy) in der klinischen Forschung ist auch die Wirkung unter Alltagsbedingungen (engl. effectiveness) zu berücksichtigen.

Das geplante Modellvorhaben sollte aus diesen Gründen folgende gleichwertige Komponenten umfassen:

1. Die Durchführung jeweils einer placebokontrollierten Studie pro Indikation.

2. Die Durchführung jeweils einer pragmatischen randomisierten Vergleichsstudie Akupunktur vs. Standardtherapie pro Indikation in einem Netz ausgesuchter Praxen.

3. Die Durchführung einer prospektiven Beobachtungsstudie, bei der Patientenmerkmale, Therapie und Verlauf aller an dem Modellvorhaben teilnehmenden Patienten sowie die Merkmale aller teilnehmenden Praxen dokumentiert werden.

4. Die Aktualisierung vorliegender systematischer Übersichtsarbeiten der klinischen Studien zur Akupunktur zu den im Modellvorhaben geprüften Indikationen.

Ad 1) Grundsätzlich wird gefordert, dass die spezifische Wirksamkeit einer Behandlung für die jeweilige Indikation wissenschaftlich belegt ist. Im Fall von Arzneimitteln erfolgt ein solcher Nachweis primär durch randomisierte, placebokontrollierte Studien. Im Fall der Akupunktur ist eine Wirksamkeit über Placebo hinaus bei den zur Diskussion stehenden Indikationen bisher nicht hinreichend gesichert. Daher erscheint es absolut sinnvoll, auch im Rahmen eines Modellvorhabens derartige Studien durchzuführen. Die Frage nach dem richtigen «Placebo» ist hier jedoch äusserst kompliziert. Die «Sham-Akupunktur» ist kein eigentliches Placebo, sondern eine reale Nadeltherapie, die aus der Sicht der Akupunkturschulen an nicht empfohlenen Körperstellen durchgeführt wird. Die existierenden Placebonadeln sind schlecht praktikabel (insbesondere Verkleben an behaarten Körperteilen), lösen ebenfalls Sensationen am Akupunkturpunkt aus (evtl. vergleichbar einer Akupressur) und sind bei Akupunkturerfahrenen Patienten kaum einzusetzen. Ein möglicher Lösungsansatz ist die zusätzliche Einführung einer Wartelistengruppe.

Ad 2) Eine Wirksamkeit über Placebo hinaus bedeutet nicht notwendigerweise, dass eine Intervention ein ähnliches oder günstigeres Nutzen/Risiko- bzw. Nutzen/Kosten-Profil als die jeweils verfügbare Standardtherapie hat. Dies ist jedoch die entscheidende Voraussetzung für eine Erstattung. Placebokontrollierte Studien sind ausserdem meist nur unter experimentellen und damit alltagsferneren Bedingungen durchführbar. Die Ergebnisse sind daher nur eingeschränkt auf die Routinepraxis übertragbar. Durch eine pragmatische multizentrische Studie in einem Netzwerk von Praxen, bei der

\section{KARGER \\ (C) 2001 S. Karger GmbH, Freiburg


Akupunktur bzw. eine leitliniengestützte Standardbehandlung mit randomisierter Zuweisung eingesetzt werden, kann dagegen unter praxisnahen Bedingungen geprüft werden, ob Nutzen (d.h. das Ausmass, inwieweit Patienten nach der Behandlung eine Besserung erfahren), Risiken (Nebenwirkungen und Komplikationen) und Kosten (inklusive Folgekosten) vergleichbar sind. Dabei ist auch die Formenvielfalt der durchgeführten Akupunkturbehandlungen zu berücksichtigen. Erweist sich die Akupunktur dabei bezüglich aller drei Aspekte als gleichwertig oder gar überlegen, so erschiene die Aufnahme in die Regelversorgung angemessen. Sollte sich bei unterschiedlichen Aspekten zum Teil eine Überlegenheit, zum Teil eine Unterlegenheit ergeben, ist abzuwägen, ob die Vorteile insgesamt die Nachteile überwiegen.

Ad 3) Auch derartige - oben genannte - pragmatische randomisierte Studien können aufgrund von begrenzten Ressourcen und organisatorischen Problemen jedoch nur einen sehr beschränkten Umfang haben. Drei wichtige Aspekte können daher weder in einer placebokontrollierten «Experimentalstudie» noch in einem pragmatischen randomisierten Praxisvergleich ausreichend untersucht werden:

a) Ein repräsentatives Abbild der Praxiswirklichkeit (wie weit sind die Ergebnisse kontrollierter Studien in die Praxis übertragbar?)

b) Sind bestimmte Patienten- und Arztmerkmale sowie unterschiedliche Akupunkturstrategien mit unterschiedlichen Prognosen assoziiert (d.h. profitieren z.B. bestimmte Patienten mehr von der Akupunktur als andere)?

c) Wie häufig treten seltene Nebenwirkungen und Komplikationen auf?

d) Wie stellt sich die Wirtschaftlichkeit der Methode in der alltäglichen Anwendungspraxis dar?

Daher ist es notwendig, mit einem angemessenen Dokumentationsaufwand die Routineversorgung mit Akupunktur und deren Ergebnisse innerhalb des Modellvorhabens zu untersuchen. Eine solche breitgefächerte Beobachtungsstudie kann ausserdem potentielle Schwachstellen in der Versorgung identifizieren, die dann mittels geeigneter Methoden des Qualitätsmanagements angegangen werden können.

Ad 4) Da im Laufe des Erprobungsvorhabens damit zu rechnen ist, dass neue Ergebnisse aus andernorts durchgeführten Studien verfügbar werden, ist es ausserdem sinnvoll, durch die Aktualisierung vorliegender systematischer Übersichtsarbeiten den Stand der Evidenz zu dokumentieren.

Dieses stufenartige Vorgehen ist im Rahmen eines allgemeinen Grundkonzepts einer Evaluation der Patientenversorgung andernorts bereits beschrieben [1] und baut in ähnlicher Form - hier in Bezug auf die Akupunktur - auf folgenden Fragen auf:

- Bei welchen Patienten wird in welcher Form und Qualität Akupunktur durchgeführt? Diese Frage zielt auf Transparenz mithilfe eines Abbilds der Praxisrealität.
- Haben die Patienten durch die Akupunktur einen medizinisch fassbaren Nutzen?

- Wie ist die Sicherheit und Verträglichkeit der Akupunktur einzuschätzen?

- Wie aufwendig ist die Akupunkturbehandlung, und können dadurch andere Kosten eingespart werden?

- Wie lässt sich die Qualität der Akupunktur verbessern?

- Wie ist die Evidenz eines spezifischen Effekts der Akupunktur zu beurteilen? (experimenteller Wirksamkeitsnachweis).

- Wie stellt sich die Evidenz der Akupunktur in der wissenschaftlichen Literatur dar? (systematische Übersichtsarbeiten).

Die vorgeschlagenen Projekte der einzelnen Stufen sollen zeitgleich realisiert werden. Durch die geplanten Massnahmen zur Prüfung der Evidenz der Akupunktur (insbesondere durch die laufende Aktualisierung der Literaturaufarbeitung) wird potenziell die Möglichkeit gegeben, bei entsprechend neuer Erkenntnislage die Laufzeit des Modellvorhabens zu verkürzen.

Eine weitere «Besonderheit» der Rahmenbedingungen der Modellversuche «Akupunktur» stellen die unterschiedlichen Interessensituationen der verschiedenen Akteure «Ärzteschaft», «Akupunkteure», «Krankenkassen» und «Patienten» dar.

Die Mehrzahl der «nicht akupunktierenden Ärzte» und ihre Standesvertreter haben kein Interesse, dass Akupunktur eine Kassenleistung wird. Dies würde den ohnehin knappen «Honorar-Kuchen» für die niedergelassenen Ärzte weiter schmälern. Die Akupunkteure selbst sind sich ebenfalls uneins. Viele befürchten, auf ihre IGEL-Leistung (individuelle Gesundheitsleistung) zukünftig verzichten zu müssen. Wo bleibt da das Patienteninteresse? Wo bleibt da die Nutzung der eigenen Chance, sich wissenschaftlich zu beweisen? Das Gejammer, nicht wissenschaftlich anerkannt zu sein oder sich im Vergleich zur konventionellen Medizin benachteiligt zu fühlen, konnte man in den vergangenen Jahrzehnten genug hören. Nun gibt es eine Chance, auf Kosten der Beitragszahler wissenschaftliche Nachweise zu erarbeiten, und plötzlich stellen sich unplausible Widerstände ein. Angenommen, die Erfahreneren unter den Akupunkteuren beteiligen sich nicht an den Modellversuchen und die Ergebnisse fallen deshalb schlecht oder schlechter aus als möglich; würde dies nicht auch erhebliche negative Auswirkungen auf Privatversicherer und Selbstzahler haben? Ein dann mit Recht selbstverschuldeter Schaden! Akupunktierende Ärzte wären gut beraten, hier mehr die Chancen als die Risiken zu sehen!

\section{Melchart, München}

\section{Literatur}

1 Melchart D, Weidenhammer W, Thormählen J, Gehring T, Saller R: Qualitätsmanagement mit Forschungsbegleitung. Z Ärztl Fortbildung QualSich (ZaeFQ) 2000;94:751-757. 